\title{
Malonyl-CoA: the regulator of fatty acid synthesis and oxidation
}

\author{
Daniel W. Foster
}

Department of Internal Medicine, University Of Texas SW Medical Center, Dallas, Texas, USA.

\begin{abstract}
In the catabolic state with no food intake, the liver generates ketones by breaking down fatty acids. During the nocturnal fast or longer starvation periods, this protects the brain, which cannot oxidize fatty acids. In 1977, we published a study in the JCI noting the surprising realization that malonylCoA, the substrate of fatty acid synthesis, was also an inhibitor of fatty acid oxidation. Subsequent experiments have borne out this finding and furthered our understanding of molecular metabolism.
\end{abstract}

Ketogenesis, the production of acetoacetate and $\beta$-hydroxybutyrate from oxidation of long-chain fatty acids, occurs only in the liver. When glucose production is insufficient, the body shifts to a lipid-based economy. However, the brain cannot oxidize fatty acids for energy, so the production of ketones is protective $(1,2)$.

It is quite remarkable how many distinguished scientists began to study ketogenesis decades ago, among them the Nobel laureates Professors H.A. Krebs and Feodor Lynen. Early on, a widespread belief was that a deficiency of oxaloacetate in the tricarboxylic acid cycle shunted acetyl-CoA derived from fatty acid oxidation into acetoacetate, as described in an excellent lecture on this history published by Professor Krebs in 1966 (3). Indeed, acetoacetate is the preferred metabolic substrate in mammalian cells, chosen above glucose, fatty acids, and other substrates $(1,2)$. However, oxidation of acetoacetate requires the enzyme succinyl-CoA:3-ketoacid-CoA transferase, which is not present in the liver; thus, the liver cannot oxidize ketones, only release them.

The fading interest in the oxaloacetate theory led to the next thought, that ketone production by the liver was dependent on the concentration of long-chain fatty acids in the blood delivered to the liver. However, utilizing the isolated perfused rat liver, we showed that oleate was not significantly converted to ketones until six hours after the initiation of fasting. This showed that there was an on/off signal in the liver to initiate and terminate ketogenesis (4).

Conflict of interest: The author has declared that no conflict of interest exists.

Citation for this article: J Clin Invest. 2012; 122(6):1958-1959. doi:10.1172/JCI63967.
My colleague Denis McGarry and I knew early that fatty acid synthesis and oxidation were reciprocal, but it was unclear how this relationship was regulated. We initially thought control was on the lipogenic side, but when we acutely blocked fatty acid oxidation, there was an immediate resumption of fatty acid and triglyceride synthesis, indicating that an inhibitor of fatty acid oxidation must be regulating the process. We were faced with two challenges: identifying the inhibition point and identifying what controlled it. To investigate this, we next compared ketogenesis from octanoic and oleic acids. We knew octanoate could penetrate the mitochondrion freely, and we found that ketogenesis from it was identical in both fed and fasted states. Octanoate oxidation, therefore, is a measure of the capacity of the $\beta$-oxidative system. Oleate, in contrast, has to be transesterified from oleyl-CoA to oleylcarnitine by the enzyme carnitine palmitoyltransferase 1 (CPT1) to enter the mitochondria. The rate of oleate oxidation increased six-fold between the fed and fasted states. Thus, we concluded that regulation was occurring at the level of CPT1 (4).

What was the inhibitor? We knew that there was a decrease in glycogen that accompanied the increase in ketogenesis. We tested dozens of molecules on CPT1, and none of them altered its activity. One morning McGarry came into the lab and said, "It has to be malonyl-CoA. It is the substrate for fatty acid synthesis, and it must also be the inhibitor." Indeed, we were able to test this directly, and the data bore out his insightful hypothesis $(5,6)$.

We knew that glucagon was the primary on signal for hepatic ketogenesis (7). Once initiated, the rate of ketone production is dependent on the level of long-chain fatty acids reaching the liver. Glucagon signaling triggers the phosphorylation and activation of AMPK. In turn, AMPK phosphorylates the two acetyl-CoA carboxylases, thereby blocking synthesis of malonyl-CoA. It simultaneously enhances destruction of malonylCoA by activating malonyl-CoA decarboxylase (Figure 1). The fall in malonyl-CoA stops fatty acid synthesis and activates CPT 1 and ketogenesis (8). We also showed that the malonyl-CoA system functions in skeletal and cardiac muscle, although these tissues do not make ketones (9).

Interestingly, we subsequently discovered that the interaction of malonyl-CoA and carnitine with CPT1 are different in liver and muscle. Inhibition of liver CPT1 requires ten times the concentration of malonyl-CoA as does the inhibition of CPT1 in the muscle and heart. Conversely, the $K_{m}$ for carnitine is much lower in liver than in muscle. These differences became important when we cloned and sequenced the liver and muscle enzymes.

The decrease in malonyl-CoA concentration is life saving during the overnight fast and, more importantly, during prolonged fasting or starvation $(1,2)$. However, it can also be deadly in uncontrolled type 1 diabetes, where markedly increased concentrations of long-chain fatty acids move the chemical state from modest ketosis to full-blown ketoacidosis if not treated (10).

A more serious problem than transient lowering of malonyl-CoA occurs in individuals who harbor genetic deficiencies in the enzymes that control carnitine levels and fat oxidation. Systemic carnitine deficiency due to a mutation in the carnitine transporter OCTN2 was the first identified cause of the syndrome of hypoketotic hypoglycemia, which can lead to sudden infant death (11). Carnitine deficiency also causes liver failure, high ammonia, cerebral edema, cardiac arrhythmias, cardiomyopathy, and muscle weakness with rhabdomyolysis.

In retrospect, the discovery of the malonyl-CoA regulatory system has had an impact far beyond the issue of ketogen- 


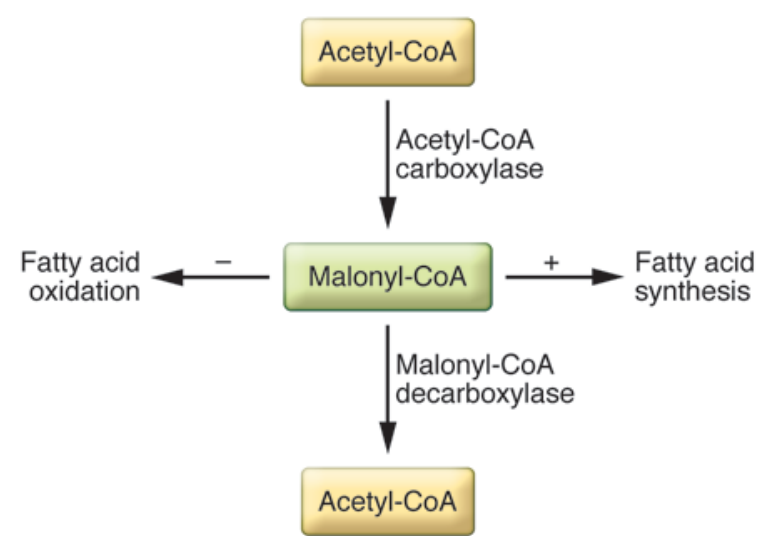

Figure 1

Regulation of malonyl-CoA determines the switch between fatty acid synthesis and oxidation. Adapted with permission from the Annals of the New York Academy of Sciences (8).

esis. The system is active in the hypothalamus, where it contributes to the regulation of food intake, in the heart, where fatty acid oxidation influences the outcome of myocardial infarction, and in the liver, where nonalcoholic steatosis may be diminished by increased fatty acid oxidation, and it is relevant in obesity, where increased mitochondrial function may cause weight loss.

I met Professor Krebs when he taught at UT Southwestern in the biochemistry course for first-year students for several years. It was remarkable and moving that he, the discoverer of the citric acid cycle, congratulated us for solving ketogenesis.

\section{Acknowledgments}

John Denis McGarry was my first fellow and longtime colleague. He died from a glioblastoma multiforme shortly after he won the Banting Medal.

Address correspondence to: Daniel W. Foster, Department of Internal Medicine, University of Texas SW Medical Center, 5323 Harry
Hines Blvd., Dallas, Texas 75390-9030, USA.

Phone: 214.648.8068; Fax: 214.648.8058;

E-mail: daniel.foster@utsouthwestern.edu.

1. Cahill GF Jr. Fuel metabolism in starvation. Annu Rev Nutr. 2006;26:1-22.

2. Cahill GF Jr, Veech RL. Ketoacids? Good medicine? Trans Am Clin Climatol Assoc. 2003;114:149-161.

3. Krebs HA. The regulation of the release of ketone bodies by the liver. Adv Enzyme Regul. 1966;4:339-53.

4. McGarry JD, Meier JM, Foster DW. The effects of starvation and refeeding on carbohydrate and lipid metabolism in vivo and in the perfused rat liver. The relationship between fatty acid oxidation and esterification in the regulation of ketogenesis. J Biol Chem. 1973;248(1):270-278.

5. McGarry JD, Mannaerts GP, Foster DW. A possible role for malonyl-CoA in the regulation of hepatic fatty acid oxidation and ketogenesis. J Clin Invest. 1977;60(1):265-270.

6. McGarry JD, Leatherman GF, Foster DW. Carnitine palmitoyltransferase 1 . The site of inhibition of hepatic fatty acid oxidation by malonyl-CoA. J Biol Chem. 1978;253(12):4128-4136.

7. McGarry JD, Wright PH, Foster DW. Hormonal control of ketogenesis: rapid activation of hepatic ketogenic capacity in fed rats by anti-insulin serum and glucagon. J Clin Invest. 1975;55(6):1202-1209.

8. Foster DW. The role of the carnitine system in human metabolism. Ann NY Acad Sci. 2004;1033:1-16.

9. McGarry JD, Woltje KF, Kuwajimia M, Foster DW. Regulation of ketogenesis and the renaissance of carnitine palmitoyltransferase. Diabetes Metab Rev. 1989;5(3):271-284

10. Foster DW, McGarry JD. The metabolic derangements and treatment of diabetic ketoacidosis. NEngl J Med. 1983;309(3):159-169.

11. Lahjouji K, Mitchell GA, Qureshi IA. Carnitine transport by organic cation transporters and systemic carnitine deficiency. Mol Genet Metab. 2001;73(4):287-297. 